\title{
Clinical Effects of Assessing Electronic Patient- Reported Outcomes Monitoring Symptomatic Toxicities During Breast Cancer Therapy - A Nationwide and Population-Based Study
}

Helle Pappot ( $\sim$ Helle.Pappot@regionh.dk)

Rigshospitalet https://orcid.org/0000-0002-3570-5372

Christina W. Baeksted

Kraeftens Bekaempelse

Aase Nissen

Kraeftens Bekaempelse

Ann Knoop

Rigshospitalet

Sandra A. Mitchell

National Cancer Institute

Jane Christensen

Kraeftens Bekaempelse

Niels Henrik Hjollund

Regionshospitalet Herning

Christoffer Johansen

Rigshospitalet

Short report

Keywords: breast cancer, patient reported outcome, symptomatic toxicities, adverse events, chemotherapy

Posted Date: August 10th, 2020

DOI: https://doi.org/10.21203/rs.3.rs-54763/v1

License: (c) (i) This work is licensed under a Creative Commons Attribution 4.0 International License. Read Full License

Version of Record: A version of this preprint was published at Breast Cancer on April 9th, 2021. See the published version at https://doi.org/10.1007/s12282-021-01244-x. 


\section{Abstract}

The involvement of cancer patients in symptom reporting talks into our cultural narrative of empowerment and participation in decisions in health of both patients and professionals. Electronic Patient Reported Outcomes (ePRO) is a tool applied for use in such interaction. Based on limited evidence and few empirical studies, health systems are rapidly implementing this instrument in managing patients in active treatment and in follow-up. In a cluster randomized trial of all Danish oncology departments treating breast cancer with adjuvant chemotherapy, we applied ePRO in 347 patients consecutively recruited. Our primary outcome, which was at least one treatment adjustment, was not significantly influenced using ePRO, 34\% in the ePRO arm and $41 \%$ in the usual care arm received at least one treatment adjustment, $p=0,095$. Number of hospitalizations and events of febrile neutropenia was not influenced by the intervention. We believe that one of the main reasons for this finding could be the application of PRO in a well-regulated treatment setting within a public health care system where the most impacting symptomatic toxicities are already taken care off.

ClinicalTrials.gov Identifier: NCT02996201, registered 19 December 2016, retrospectively registered.

\section{Short Report}

Information concerning symptomatic toxicities leading to treatment adjustments are currently being investigated applying a range of Patient Reported Outcome (PRO) instruments. The introduction of such measures acknowledges the discrepancy between symptoms reported by patients and clinicians (1-3). Promising results regarding symptom control $(4,5)$ and even survival $(6,7)$ have highlighted the potential clinical benefits based on regular registration of ePRO. However, studies published do have limitations by including highly selected, health-insured patients (6) or quite heterogeneous patients in terms of stage of disease at time of inclusion (7) reducing generalizability of the findings. Despite the preliminary and limited evidence, ePRO is currently being implemented across many health systems in different settings. Before publication of the landmark trials by Basch and Denis $(6,7)$ we planned, registered and initiated a nationwide, population-based randomized clinical trial examining the impact of applying ePRO in a public health system. We aimed at reducing treatment adjustments and hospitalizations, in breast cancer patients cluster-randomized immediately before initiating six cycles of standard adjuvant chemotherapy.

All 11 Danish departments treating breast cancer patients with adjuvant chemotherapy (8) (9) took part in the study. Departments were randomized either to let the patients use an electronic PRO questionnaire with real-time feedback to clinicians (ePRO arm) for reporting of symptom toxicity during chemotherapy or to use standard methods for handling of symptomatic toxicities. Stratification for department size and geography was applied. The ePRO presented 42 PRO-CTCAE questions $(10,11)$ on 25 symptoms to the patients (12). At departments randomized to use ePRO (Fig. 1a), patients completed a questionnaire on a tablet computer before each cycle of chemotherapy and current and past reporting was presented to the clinicians (13). Reviewing of data was not mandatory for the health professionals. 
Primary endpoint was number of patients with one or more treatment adjustment; secondary endpoints were number of patients with one or more hospitalizations and one or more events of febrile neutropenia. Number of patients with treatment postponed more than seven days as well as completion of the scheduled six cycles of chemotherapy were registered. Compliance to ePRO was measured by the number of answered questionnaires. The study had a power of $80 \%$ to detect a difference of 15 percentage points for ePRO arm versus usual care arm for the treatment adjustment, which has been reported to occur in approximately $50 \%$ of patients (40-62\%) (14) and 5 percentage points (10 to $5 \%$ ) for the secondary outcomes. For this purpose, 700 patients were needed. A generalized linear mixed model analysis was performed for each of the three binominal endpoints adjusting for age and treatment regimen using SAS 9.4

Of 682 patients enrolled, 347 (ePRO arm) and 335 (usual care arm) (Fig. 1b), the median age was 53 years in both arms (range 21-82). Baseline variables were well balanced, apart from treatment regimens. In total, $34 \%$ in the ePRO arm and $41 \%$ in the usual care arm received at least one treatment adjustment; however, this difference was not statistically significant (Fig. 2a). No difference was found regarding hospitalization or febrile neutropenia (Fig. 2a).

In the ePRO arm, $32 \%$ had at least one chemotherapy cycle postponed ( $\geq 7$ days), compared to $38 \%$ in the usual care arm (Fig. 2b). The six scheduled cycles of chemotherapy were completed without treatment adjustments in 66\% (ePRO arm) and 59\% (usual care arm) (Fig. 2b).

Compliance in the ePRO arm was $87 \%$, as patients answered 1,747 questionnaires out of 2,015 possible. $63 \%$ in the ePRO arm had the completions of the electronic PRO questionnaire reviewed by a health professional at least once.

Contrary to our expectations, we did not find a significant effect on our main outcomes. We carried out the study in a tax-paid, public health system, free of charge for every citizen implying that patients fragile due to existing comorbidity, social status and psychological functioning are a part of the population admitted to the oncology clinic. Despite the non-significant character of our findings, the overall results point in the direction of clinical value as previously reported in a population of metastatic cancer patients from the US (6) and a population of French lung cancer patients (7).

The fact, that only a non-significant effect of the intervention was observed may be influenced by two conditions. First, the intervention might be of low potency compared to usual practice if the most impacting symptomatic toxicities are already taken care off. This consideration arises due to the content of the high-quality treatment program for breast cancer patients following Danish national guidelines and the tax-paid public health system, www.DBCG.dk. Second, the study was underpowered to detect an existing difference between the study arms, as the frequency of dose adjustments (34-40\%) in both study arms turned out to be less than anticipated (50\%) when the study was planned. Number of hospitalizations was high in both study arms, however this excess number of hospital events was also reported in a large cohort study of more than 8,000 breast cancer women in Canada (15). Due to existing workload we were not able to include mandatory review of patients' PRO reporting, which is reflected in 
the number of reviews. This is a weakness, since improved patient-clinician communication is needed for better management of symptomatic adverse events, and the study would have benefited from a more detailed analysis of this issue. Further, in our study report of severe symptoms did not automatically alerted the providers for further intervention. In this context the establishment of clinical guidelines, which outline mandatory review by health professionals of PRO information and guidance for intervention would possibly influence the effect of implementing PRO during treatment of a breast cancer patients.

To the best of our knowledge, we report the first randomized trial assessing a PRO instrument among cancer patients in active treatment following the two reports by Basch and Denis, respectively. We find no significant of using ePRO on our primary outcome and ascribe this result to several methodological issues within the study design and execution. The effect of implementation of ePRO in oncology may be highly dependent on the setting with respect to e.g. diagnosis, stage of disease, treatment type, population characteristics and health care system.

\section{Declarations}

\section{Ethic approval and consent to participate}

Ethics approval for the present study was not needed due to Danish law. The study was approved by the Danish Data Protection Agency (File number, 2015-41-4190) and registered at Clinicaltrials.gov (Clinicaltrials.gov identifier NCT02996201). All participants included completed informed consent.

\section{Consent for publication}

Not applicable

\section{Availability of data and materials}

The dataset used during the current study is available from the corresponding author on reasonable request.

\section{Competing interest}

The authors declare no competing interests in relation to the present publication.

\section{Funding}

This study was funded by the Danish Cancer Society (R113-A7084-14-S34). 


\section{Authors contribution}

$\mathrm{HP}, \mathrm{CWB}, \mathrm{AaN}$ and $\mathrm{CJ}$ designed the study and planned the implementation with $\mathrm{AK}$. NHH designed the software. HP and CWB performed the study. CWB and JC performed the statistical analysis. All authors were actively involved in interpreting the results and writing the manuscript.

\section{Acknowledgements}

The authors wish to thank all breast cancer patients who took part in this study.

\section{List Of Abbreviations}

PRO - Patient Reported Outcomes

ePRO - electronic Patient Reported Outcomes

\section{References}

1. Di Maio M, Gallo C, Leighl NB, Piccirillo MC, Daniele G, Nuzzo F, et al. Symptomatic Toxicities Experienced During Anticancer Treatment: Agreement Between Patient and Physician Reporting in Three Randomized Trials. J Clin Oncol. 2015.

2. Falchook AD, Green R, Knowles ME, Amdur RJ, Mendenhall W, Hayes DN, et al. Comparison of Patient- and Practitioner-Reported Toxic Effects Associated With Chemoradiotherapy for Head and Neck Cancer. JAMA Otolaryngol Head Neck Surg. 2016;142(6):517-23.

3. Chera BS, Amdur RJ, Tepper J, Qaqish B, Green R, Aumer SL, et al. Phase 2 Trial of De-intensified Chemoradiation Therapy for Favorable-Risk Human Papillomavirus-Associated Oropharyngeal Squamous Cell Carcinoma. Int J Radiat Oncol Biol Phys. 2015;93(5):976-85.

4. Bruner DW, Movsas B, Basch E. Capturing the patient perspective: patient-reported outcomes as clinical trial endpoints. Am Soc Clin Oncol Educ Book. 2012:139-44.

5. Howell D, Molloy S, Wilkinson K, Green E, Orchard K, Wang K, et al. Patient-reported outcomes in routine cancer clinical practice: a scoping review of use, impact on health outcomes, and implementation factors. Ann Oncol. 2015;26(9):1846-58.

6. Basch E, Deal AM, Dueck AC, Scher HI, Kris MG, Hudis C, et al. Overall Survival Results of a Trial Assessing Patient-Reported Outcomes for Symptom Monitoring During Routine Cancer Treatment. JAMA. 2017;318(2):197-8.

7. Denis F, Basch E, Septans AL, Bennouna J, Urban T, Dueck AC, et al. Two-Year Survival Comparing Web-Based Symptom Monitoring vs Routine Surveillance Following Treatment for Lung Cancer. JAMA. 2019;321(3):306-7. 
8. Roche H, Fumoleau P, Spielmann M, Canon JL, Delozier T, Serin D, et al. Sequential adjuvant epirubicin-based and docetaxel chemotherapy for node-positive breast cancer patients: the FNCLCC PACS 01 Trial. J Clin Oncol. 2006;24(36):5664-71.

9. Sparano JA, Zhao F, Martino S, Ligibel JA, Perez EA, Saphner T, et al. Long-Term Follow-Up of the E1199 Phase III Trial Evaluating the Role of Taxane and Schedule in Operable Breast Cancer. J Clin Oncol. 2015;33(21):2353-60.

10. Basch E, Reeve BB, Mitchell SA, Clauser SB, Minasian LM, Dueck AC, et al. Development of the National Cancer Institute's patient-reported outcomes version of the common terminology criteria for adverse events (PRO-CTCAE). JNatlCancer Inst. 2014;106(9).

11. Baeksted C, Nissen A, Pappot H, Bidstrup PE, Mitchell SA, Basch E, et al. Danish Translation and Linguistic Validation of the U.S. National Cancer Institute's Patient-Reported Outcomes version of the Common Terminology Criteria for Adverse Events (PRO-CTCAE). J Pain Symptom Manage. 2016;52(2):292-7.

12. Pappot HBC, Knoop A, Mitchell SA, Nissen A, Johansen C. Routine surveillance for symptomatic toxicities with real-time clinician reporting in Danish breast cancer patients-Organization and design of the first national, cluster randomized trial using the Patient-Reported Outcomes version of Common Terminology Criteria for Adverse Events (PRO-CTCAE). Breast J. 2019 Mar;25(2):269-72. doi:10.1111/tbj.13204. Epub 2019 Feb 1.

13. Hjollund NH, Larsen LP, Biering K, Johnsen SP, Riiskjaer E, Schougaard LM. Use of Patient-Reported Outcome (PRO) Measures at Group and Patient Levels: Experiences From the Generic Integrated PRO System, WestChronic. InteractJMedRes. 2014;3(1):e5.

14. Bhatnagar B, Gilmore S, Goloubeva O, Pelser C, Medeiros M, Chumsri S, et al. Chemotherapy dose reduction due to chemotherapy induced peripheral neuropathy in breast cancer patients receiving chemotherapy in the neoadjuvant or adjuvant settings: a single-center experience. SpringerPlus. 2014;3:366.

15. Enright K, Grunfeld E, Yun L, Moineddin R, Ghannam M, Dent S, et al. Population-based assessment of emergency room visits and hospitalizations among women receiving adjuvant chemotherapy for early breast cancer. J Oncol Pract. 2015;11(2):126-32.

\section{Figures}


A

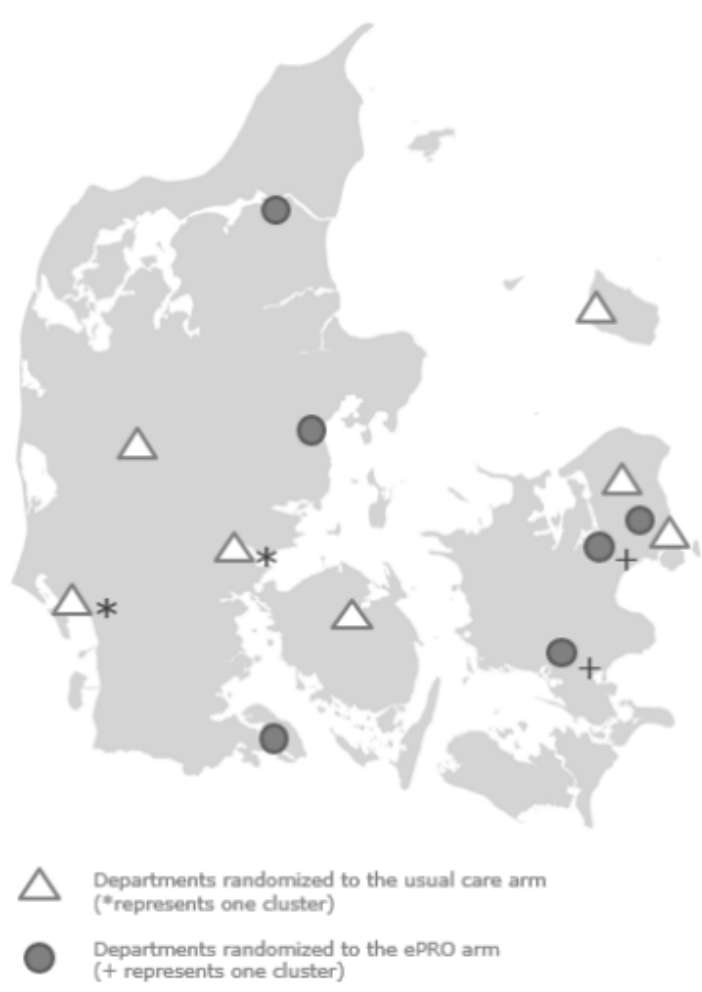

B

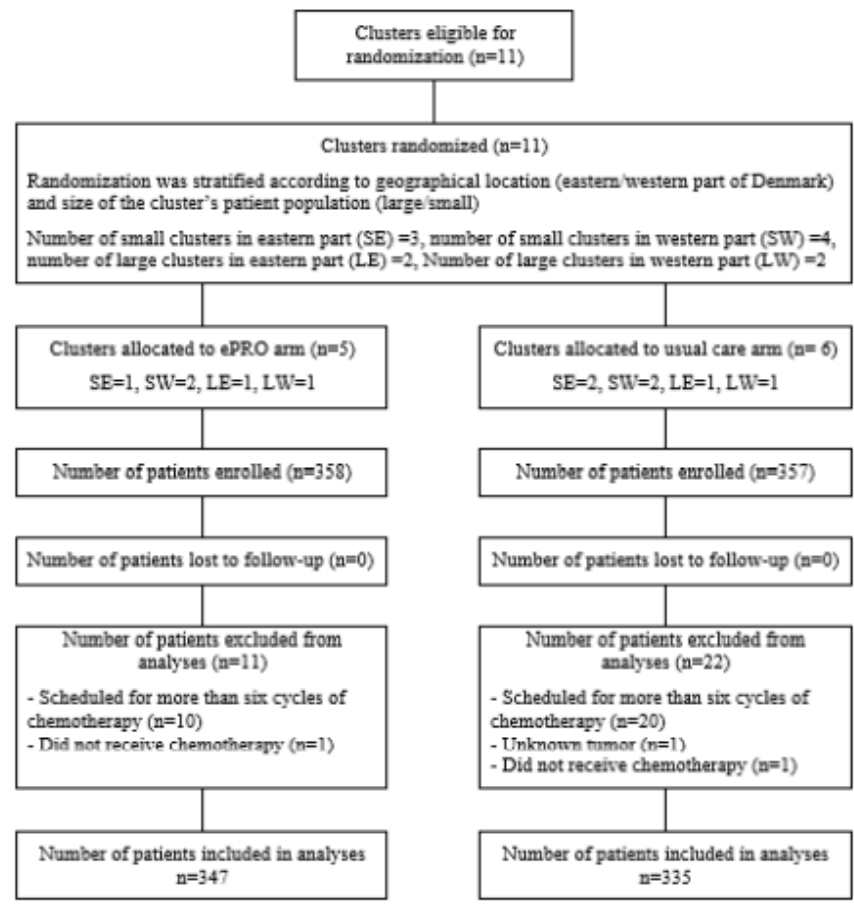

\section{Figure 1}

Randomization. A) Results of the cluster randomization stratified for geographic location and size of the cluster's patient population. B) CONSORT diagram of the randomization process.

A

\begin{tabular}{|c|c|c|c|}
\hline & $\begin{array}{r}\text { ePRO } \\
(\mathbf{n}=\mathbf{3 4 7}) \\
\mathbf{n} \\
(\%)\end{array}$ & $\begin{array}{r}\text { Usual } \\
\text { care } \\
(\mathbf{n}=\mathbf{3 3 5}) \\
\mathbf{n} \\
(\%)\end{array}$ & $\begin{array}{r}\text { Odds Ratio (OR) for } \\
\text { patients in the ePRO arm } \\
\text { compared to the usual care } \\
\text { arm }^{1}\end{array}$ \\
\hline $\begin{array}{l}\text { Treatment } \\
\text { adjustments }^{2}\end{array}$ & $\begin{array}{r}118 \\
(34.0)\end{array}$ & $\begin{array}{r}136 \\
(40.6)\end{array}$ & $\begin{array}{r}\mathrm{OR}=0.75,95 \% \mathrm{CI}: 0.54-1.05, \\
\mathrm{p}=0.095^{3}\end{array}$ \\
\hline Hospitalization & $\begin{array}{r}89 \\
(25.6)\end{array}$ & $\begin{array}{r}75 \\
(22.4)\end{array}$ & $\begin{array}{r}\mathrm{OR}=0.90,95 \% \text { CI: } 0.60-1.35, \\
\mathrm{p}=0.616^{3}\end{array}$ \\
\hline $\begin{array}{l}\text { Febrile } \\
\text { neutropenia }\end{array}$ & $\begin{array}{r}31 \\
(8.9)\end{array}$ & $\begin{array}{r}35 \\
(10.4)\end{array}$ & $\begin{array}{r}\mathrm{OR}=0.72,95 \% \text { CI: } 0.40-1.28, \\
\mathrm{p}=0.257^{3}\end{array}$ \\
\hline
\end{tabular}

*Number of patients with minimum one event of febrile neutropenia/hospitalization/ treatment adjustment during six cycles of chemotherapy

${ }^{1} \mathrm{OR}$ for having at least one event of treatment adjustment/hospitalization/febrile neutropenia ${ }^{2}$ Dose reduction or change of treatment regimen

${ }^{3} \mathrm{~A}$ generalized linear mixed model taking into account the cluster randomization was used.

Models were adjusted for age and treatment regimen
B

\section{$\%$ of patients}

100

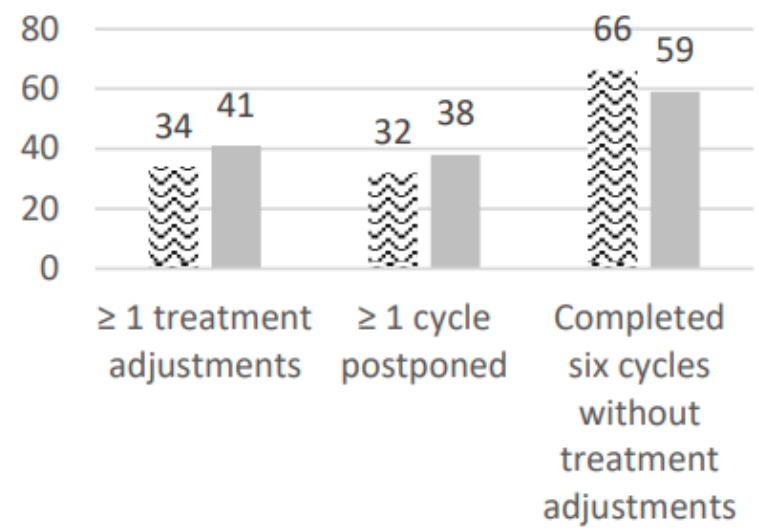

¿PRO arm Usual care arm 


\section{Figure 2}

Results. A) Primary results regarding treatment adjustments, hospitalization and febrile neutropenia. B) Descriptive results regarding treatment adjustments, postponement of chemotherapy and completion of full treatment schedule. 